EPJ Web of Conferences 52, 03002 (2013)

DOI: $10.1051 /$ epjconf/20135203002

C) Owned by the authors, published by EDP Sciences, 2013

\title{
Collimation of energetic neutrinos and muons inside EAS cores
}

\author{
Jean-Noël Capdevielle ${ }^{1, a}$, Mohammed Cherif Talai ${ }^{2}$, and Reda Attallah ${ }^{2}$ \\ ${ }^{1}$ APC Université Paris Diderot, 10 rue A. Domon et V. Duquet, 75013 Paris, France \\ ${ }^{2}$ Département de Physique, Université Badji Mokhtar, B. P. 12, 23000 Annaba, Algeria
}

\begin{abstract}
High-energy muon and neutrino components are simulated inside cores of EAS generated by primary protons and heavy nuclei of energies $10^{14}-10^{20} \mathrm{eV}$. The collimation of muons and neutrinos inside the core is compared with present penetrating beams generated by accelerators.
\end{abstract}

\section{Introduction}

Near 3 decades ago De Rujula, Glashow, Wilson and Charpak [1] advocated the employment in CERN of a new generation of proton synchrotron to explore the Earth with neutrino beams. They pointed out the necessity to focus the pion beam produced in the original proton interactions with adequate magnets. Nowadays several facilities are able to propagate inside the Earth crust neutrino beams after the decay of charged pions and kaons in tunnels of suitable length following the pion collimation with magnetic horns. Such is the case of KEK J. PARC and T2K (250 and $395 \mathrm{~km}$ to Kamiokande), OPERA and MINOS (732 km to Gran Sasso and Irvine Mine, resp.).

In order to observe in cosmic rays properties helpful for a future exploration of the Earth, we have performed simulations intended to identify inside the EAS cores the natural conditions of collimation of pion beams able to replace the magnetic focusing. The attention is concentrated on muon and neutrino features close to the shower axis (lateral distributions, energy spectrum, angular distributions). Vertical as well as near horizontal showers are produced to understand the behavior of the beams during terrestrial underground long path up to $12300 \mathrm{~km}$ or shorter than $1000 \mathrm{~km}$ with the present technology.

Even if $10^{6}$ neutrinos can be contained in a radius of $2 \mathrm{~m}$ around the core of a giant shower of $10^{20} \mathrm{eV}$ energy (as suggested hereafter by comparable densities of muons on fig. 4), the signal carried by upward detectable neutrinos is not comparable with the pulse of an accelerator. However, some visible signatures might happen during particular atmospheric conditions (thunderstorms, electrostatic unbalanced state between clouds and upper atmospheric layers) induced by ascending ion channels

\footnotetext{
a e-mail: capdev@apc.univ-paris7.fr
}

produced in the atmosphere by cascades emerging from the ground.

We have to remember here that upgoing showers were considered half a century ago [2], as well as slightly later horizontal air showers (HAS). The interest of HAS to detect diffuse neutrinos appeared limited by the prompt muon production but the interest for upward EAS was underlined again in 1983-84 [3,4] and detailed calculations were also performed [5, 6]. One detailed synthesis of neutrino's path in the Earth and upward going muons was described [7].

For the purpose of comparing the rare signals which could be recorded in different circumstances with walls of emulsion bricks, acoustic detectors, large area muon detectors at Earth surface and optical detectors for upgoing showers, we have carried out extensive simulations. Our results concerning the general features of the penetrating component near the EAS centre are reported in the next section, whereas the collimation of the neutrino beam in EAS cores is compared to synchrotron generated beam in the following section.

\section{The penetrating component at ground level}

\subsection{Simulations with CORSIKA}

We have selected the version CORSIKA 6.617 [8] with QGSJET model describing the multiple production generating via charged pion and kaon decays the most important part of muons and neutrinos reaching ground level. CORSIKA options involved in the simulation are the following:

- Hadronic interaction model: QGSJET.

- Primary particle: proton, iron.

- Primary particle energy : $10^{5}$ up to $10^{9} \mathrm{GeV}$.

- Analysis concentrated on : neutrinos and muons. 


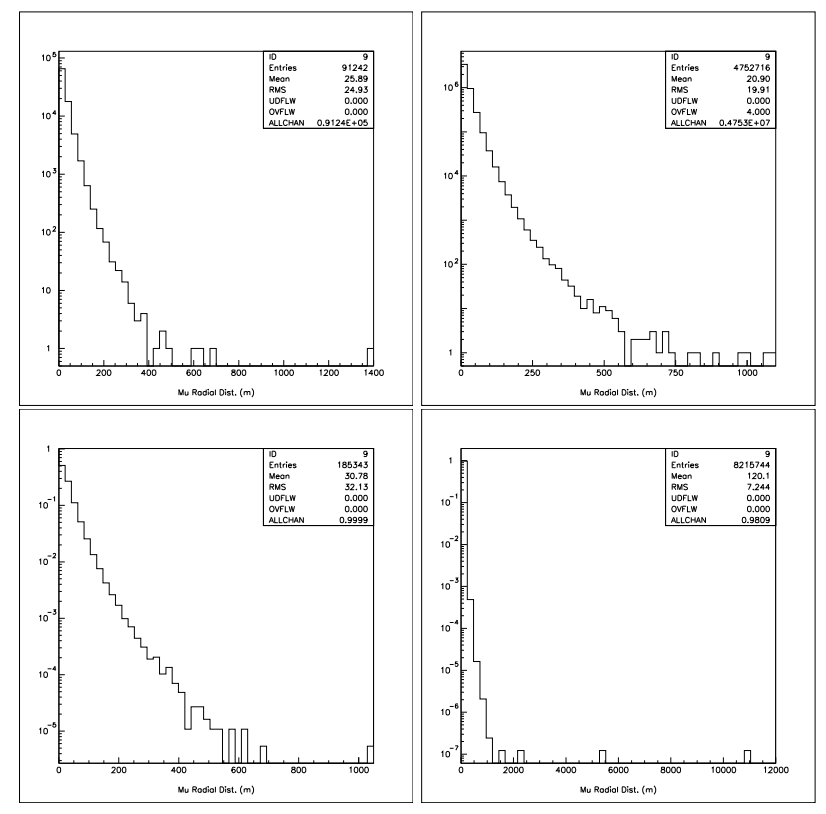

Figure 1. Lateral muon densities for $\mathrm{p}$ and Fe primaries, $10^{16}$, and $10^{18} \mathrm{eV}$.

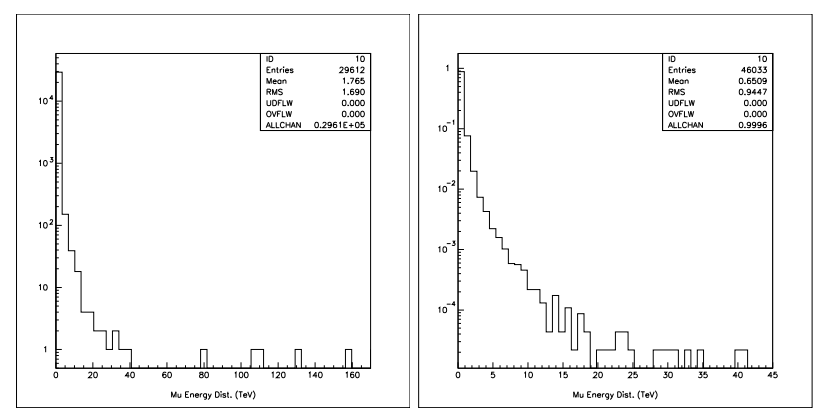

Figure 2. Energy distributions for $\mathrm{p}$ and Fe primaries, $E_{0}=$ $10^{16} \mathrm{eV}$.

- Energy cutoff : $100 \mathrm{GeV}$.

- EAS core : radius $\leq 10 \mathrm{~m}$.

The choice of a common lower energy threshold of $100 \mathrm{GeV}$ (or above) facilitates the observation of possible features correlated to the primary interaction properties.

The conditions of simulations performed in addition for giant EAS [9] up to $10^{20} \mathrm{eV}$ have been adapted with adequate thinning parameters. At present, the data analysis has been performed only for the muon component above $10^{19} \mathrm{eV}$.

\subsection{Energetic muons in EAS core}

We have first compared the lateral distribution of positive and negative muons obtained for proton and iron primaries on Fig. 1 at $10^{7}$ and $10^{9} \mathrm{GeV}$. The energy distributions are shown on Fig. 2 for $\mathrm{p}$ and Fe, respectively. The energy sum contained in EAS cores are also shown on Fig. 3. The very large densities of muons obtained in giant showers are shown on Fig. 4.

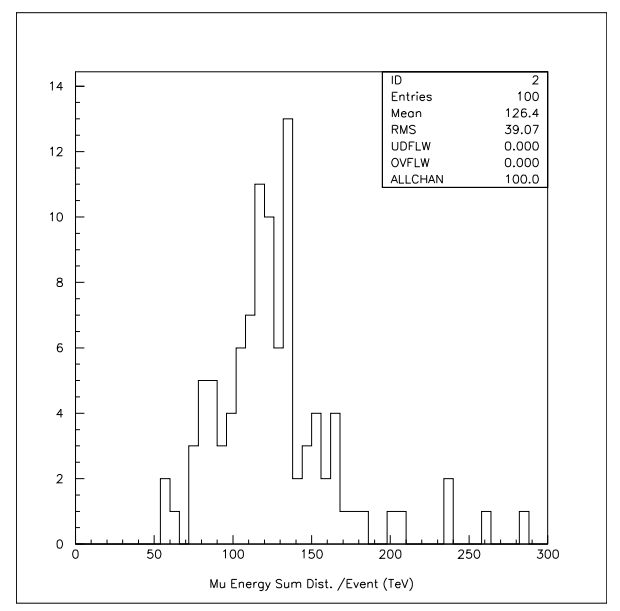

Figure 3. Energy Sum, $E_{0}=10^{16} \mathrm{eV}$.

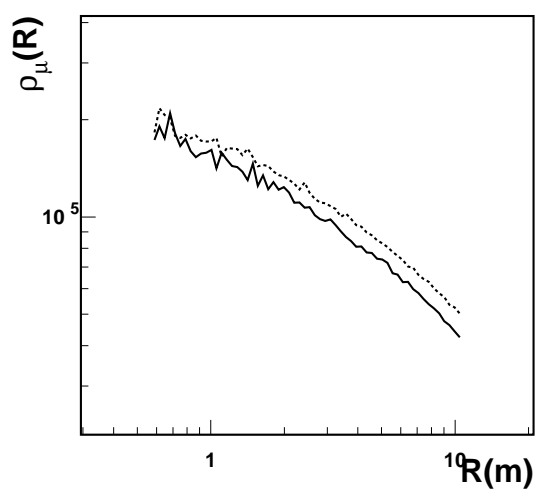

Figure 4. Lateral muon densities for $\mathrm{p}$ and $\mathrm{Fe}$ primaries, $E_{0}=$ $10^{20} \mathrm{eV}$.

Table 1. Neutrino multiplicity for different circumstances.

\begin{tabular}{llll}
\hline$E_{0}(\mathrm{GeV})$ & $10^{5}$ & $10^{7}$ & $10^{9}$ \\
\hline $\mathrm{p}$ & 1.92 & 115 & 6990 \\
$\mathrm{Fe}$ & 0.75 & 196 & 10730 \\
\hline
\end{tabular}

\subsection{Energetic neutrinos in EAS core}

In parallel the different neutrino energy and radial distributions are presented in one case (Fig. 7). We have also given in table 1 neutrino multiplicities in several circumstances. The neutrino size $\left(E_{v} \geq 100 \mathrm{GeV}\right)$ is presented up to ultra high energy for $\mathrm{p}$ and Fe primaries on Fig. 5.

The very high energy neutrino content is plotted versus primary energy for thresholds 10 and $100 \mathrm{TeV}$, for $\mathrm{p}$ and Fe primaries combined with zenith angles of $0^{\circ}$ and $70^{\circ}$ on Fig. 6. 
Neutrino multiplicity N 2 above $100 \mathrm{GeV}$ )

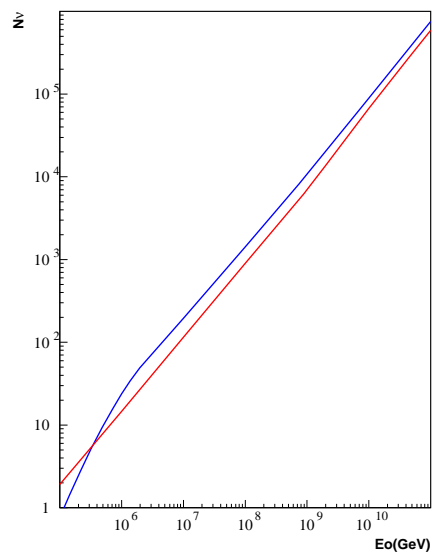

Figure 5. Neutrino contents in EAS core up to $E_{0}=10^{20} \mathrm{eV}$, upper curve Fe primaries, lower curve p primaries, vertical incidence.

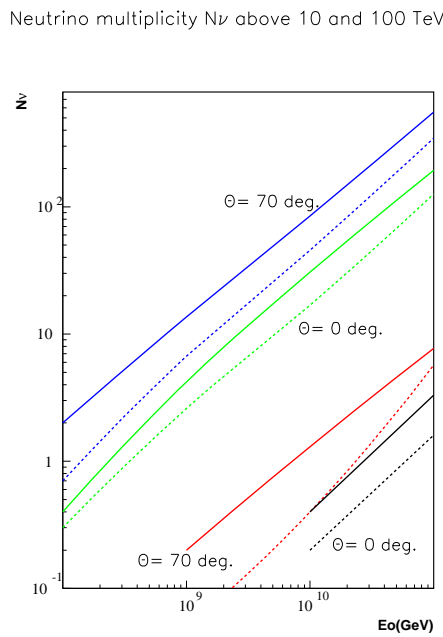

Figure 6. Very high energy neutrino contents (thresholds $10 \mathrm{TeV}$, $100 \mathrm{TeV}$ ) for for $\mathrm{p}$ and $\mathrm{Fe}$ primaries up to $E_{0}=10^{20} \mathrm{eV}$, inclinations $0^{\circ}, 70^{\circ}$.

\section{Collimation efficiency in accelerators beams and in EAS cores}

\subsection{Electromagnetic focusing with synchrotron beams}

Super magnets for focusing pion beams, decaying in $\mathrm{km}$ tunnels, permit nowadays the propagation of neutrinos beams from proton synchrotron in the Earth crust at distances of $700-1000 \mathrm{~km}$. The pocket formula of the pioneers (as seen in Sect. 1) [1] illustrates clearly the problem in distinguishing the two sources of angular divergence, the angle of emission of secondary mesons (mainly charged pions) $\Theta_{p-\pi}$, and the angle of emission of the muon-neutrino during pion decay) $\Theta_{\pi-v}$ :
Table 2. Average zenith angle in degrees of $v$ 's, sea level, vertical shower

\begin{tabular}{lllll}
\hline$E_{0}(\mathrm{GeV})$ & $10^{5}$ & $10^{6}$ & $10^{7}$ & $10^{8}$ \\
\hline $\mathrm{p}$ & 0.024 & 0.032 & 0.036 & 0.039 \\
$\mathrm{Fe}$ & 0.025 & 0.023 & 0.028 & 0.033 \\
\hline
\end{tabular}

$$
\begin{gathered}
\Theta_{p-\pi} \sim 0.3 \mathrm{GeV} /<E_{\pi}>\sim 1.2 \times 10^{-4}\left(10 \mathrm{TeV} / E_{p}\right) \\
\Theta_{\pi-v} \sim 0.03 \mathrm{GeV} /<E_{v}>\sim 0.5 \times 10^{-4}\left(10 \mathrm{TeV} / E_{p}\right)
\end{gathered}
$$

Thanks to a good magnetic focusing of the charged pion such that $\Theta_{p-\pi} \ll \Theta_{\pi-v}$, the overall divergence is governed by the neutrino decay angle. Consequently, the radius of the neutrino beam of a $10 \mathrm{TeV}$ accelerator was expected $50 \mathrm{~m}$ for a distance of $1000 \mathrm{~m}$. This radius is about $2 \mathrm{~km}$ for OPERA after $732 \mathrm{~km}$ of propagation[10] from the $400 \mathrm{GeV}$ CERN Super-Synchrotron with lower neutrino energy (about $18 \mathrm{GeV}$ ).

\subsection{Natural focusing by relativistic dynamics}

The ratio $p_{\mathrm{t}} / p_{\text {long }}$ in laboratory system governs the concentration of high energy muons and neutrinos close to shower axis and the angle $\Theta_{p-\pi}$ or $\Theta_{\pi-\pi}$ remains very small as far as we are dealing with muon-neutrino fluxes of energies exceeding $100 \mathrm{GeV}$ and primary comic ray energies the upper energy limit attained now by the LHC.

This is illustrated by the angular distributions calculated with CORSIKA together for proton and iron primaries as shown in Fig. 7. The average neutrino emission angle of the neutrinos with respect to the shower axis is given in the following table 2 for different primaries and energies.

\subsection{Consequences for fluxes of neutrinos penetrating deeply in the Earth}

In order to appreciate the propagation of the $v_{\mu}$ and $\overline{v_{\mu}}$ inside the Earth crust, we have employed for the mean free path $\Lambda_{v}$ (in $\mathrm{km}$ ) the following expression [11]:

$$
\Lambda_{v}=600\left(\rho_{\text {rock }} / 2.65 \mathrm{~g} / \mathrm{cm}^{3}\right)^{-1}\left(\sigma_{v} / 10^{-32} \mathrm{~cm}^{-2}\right)^{-1}
$$

$\sigma_{v}$ being the neutrino cross section. A very simplified profile of the Earth as in [1] has been taken as indicated herafter:

- Density core $10 \mathrm{~g} \mathrm{~cm}^{-3}$; Diameter $6800 \mathrm{~km}$.

- Mantle $5.5 \mathrm{~g} \mathrm{~cm}^{-3}$; Thickness $4000 \mathrm{~km}$.

- Crust $3.5 \mathrm{~g} \mathrm{~cm}^{-3}$; Thickness $1956 \mathrm{~km}$.

- Total (vertical crossing) $12700 \mathrm{~km}$.

- Total (inclined crossing $\theta=28^{\circ}$ ) $11263 \mathrm{~km}$ off core.

For very high energies, the mean free path of the neutrinos (here neutrino-muons and antineutrino-muons taken simultaneously) decreases rapidly in reason of the rise of $\sigma_{v}$ versus primary energy taken following [7]. The dependance is shown in table 3 in different sections of the Earth. 


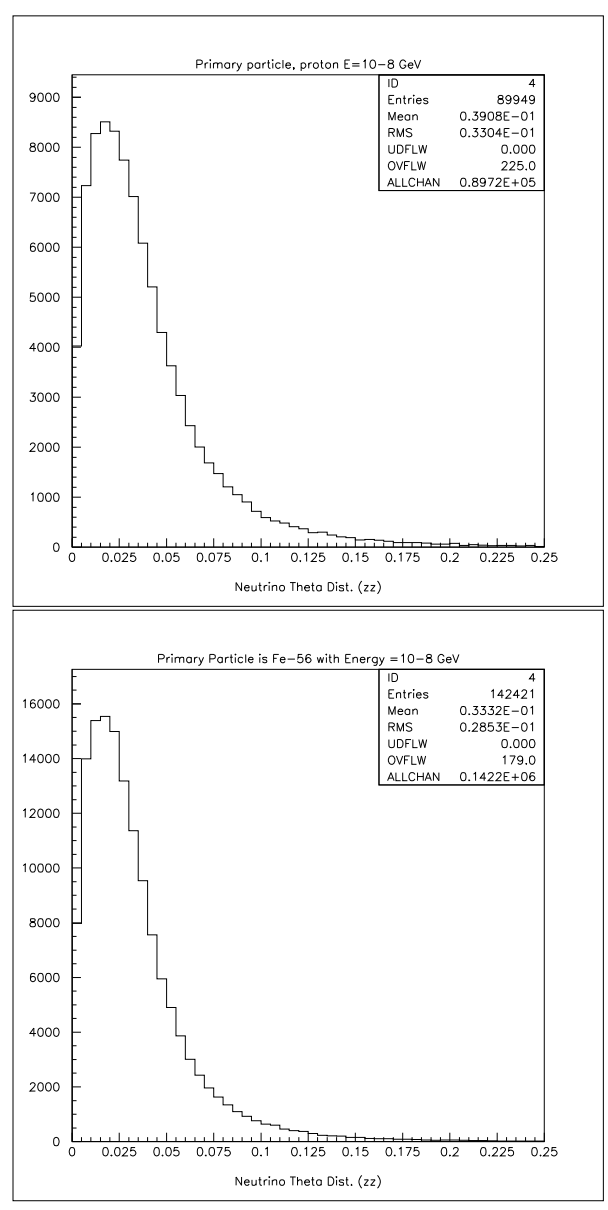

Figure 7. Angular neutrino distribution for $\mathrm{p}$ and Fe primaries, $E_{0}=10^{17} \mathrm{eV}$.

Table 3. $v$ 's mean free path in Earth in km (first line in million $\mathrm{km})$.

\begin{tabular}{llll}
\hline$E_{v}(\mathrm{TeV})$ & core & mantle & crust \\
\hline 0.1 & 26.5 & 48.1 & 75.7 \\
1.0 & 265000 & 481818 & 757442 \\
5.0 & 64810 & 117832 & 185172 \\
10. & 35338 & 64251 & 100966 \\
50. & 14579 & 26508 & 41656 \\
100. & 9958 & 18105 & 28451 \\
200. & 6804 & 12366 & 19432 \\
\hline
\end{tabular}

The nadir dependance appears on table 4 where the attenuation of neutrino fluxes is calculated for different angles $\Theta=0^{\circ}, 28^{\circ}$ corresponding respectively to trajectories passing by the Earth centre or tangential crossing to the core. $I_{v}\left(28^{0}\right) / I_{v}\left(0^{0}\right)$ represents the ratio to the vertical transport. The discrepancy for fixed $E_{v}$ turns to a double abundance for $E_{v} \sim 300 \mathrm{TeV}$.

With a similar model for the internal structure of the earth [12], a comparable enhancement of the flux transmitted at an incidence $\Theta=33^{0}$ instead of $\Theta=0^{0}$ : such circumstances suggest that the comparison of the ratio of upward neutrino fluxes near $30^{\circ}$ to vertical ascending fluxes could be an indicator of the internal earth structure.

We note also that the ascending showers initiated in the at-
Table 4. Neutrino fluxes attenuation inside the Earth versus $E_{v}$.

\begin{tabular}{llll}
\hline$E_{v}(\mathrm{TeV})$ & $0^{0}$ & $28^{0}$ & $I_{\nu}(28) / I_{\nu}(0)$ \\
\hline 0.1 & 0.9963 & 0.9978 & 1.0014 \\
1.0 & 0.964 & 0.978 & 1.0146 \\
5.0 & 0.861 & 0.913 & 1.069 \\
10. & 0.760 & 0.847 & 1.146 \\
50. & 0.514 & 0.669 & 1.301 \\
100. & 0.378 & 0.555 & 1.469 \\
200. & 0.240 & 0.473 & 1.757 \\
\hline
\end{tabular}

mosphere after the transfer of energetic astrophysical neutrinos through the globe might conserve the direction of the source taking into account moderate deviations of energetic neutrinos exhibited in fig. 7, $\Theta_{p-\pi}, \Theta_{\pi-\nu}$.

This is again the case of the deviations occurring at the emergence at the antipodes such as for instance in the case of hadron production $v+N->\mu+$ Hadrons considered previously[12] where

$$
\Theta_{v \mu} \sim 2.6\left(0.1 T e V / E_{\mu}^{0.5}\right)
$$

Hence, the measurement of the angular dependance of HE neutrinos emerging inside a solid angle with an aperture of $40^{\circ}$ around the nadir appears as an approach of the border between the central solid core and its liquid environment inside the earth.

\section{Further investigations with HE neutrinos and muons}

A preliminary estimation from our limited data base suggests that about 50 to 100 giant showers generated by protons of energy of $10^{20} \mathrm{eV}$ would be necessary to generate a single upward neutrino of $200 \mathrm{TeV}$ emerging along the shower axis at the antipode. A few Monte Carlo simulations in the upward direction testified up to $20 \mathrm{~km}$ altitude have shown us that the end of a cascade is characterized by a long tail with a moderate absorption. We have not yet estimated how such shower could initiate a ionization channel around the axis between areas negatively charged inside the clouds at an altitude of $2 \mathrm{~km}$ and atmospheric slides in the stratosphere containing positive charged areas above $20 \mathrm{~km}$; the detail of the consequences of the multiple coulomb scattering during the propagation through low atmospheric densities and of the deviations due to the magnetic field needs longer calculations ; however, such circumstance can be a candidate to help the understanding of the initiation of lightnings and transient lumineous events (TLE) with tremendous electric discharges.

Recently a group of five gigantic jets climbing up to $90 \mathrm{~km}$ altitude has been observed with unpreceeding details above an isolated tropical storm east Réunion Island [13]. The large number of downward showers, taking into account the area of the island (area $2512 \mathrm{~km}^{2}$ ), about one third covered by the storm environment, can be taken as a first candidate but those showers are not able to generate ionisation at high altitude around the shower axis.bIn the case of upward vertical showers generated by remnants of giant EAS propagated along an earth diameter 
for a near vertical emergence the number of showers of AUGER recorded in one hour on an area comparable to the island indicate that we are several under orders of magnitude under to produce the required flux of showers going upward. This gives small chances to explain transient luminar events with giant lightning related to long ionisation channels developed in the high atmosphere by energetic ascending showers generating discharges between atmospheric slides with different potential. Furthermore our study is far to be complete, for instance the delayed effects of the neutrons around the shower axis have not been considered.

The case of muons above $10 \mathrm{TeV}$ and even $100 \mathrm{TeV}$ (in the core of downwards EAS) looks more interesting in the case of inclined EAS as far as they can be identified in sophisticated neutrino detectors. Those muons appear under the form of families conserving a few hints of the earliest interactions, especially tranverse momentum distribution, discrepancies between showers initiated by p or heavy nuclei.

An average number of muons around 5 (energies exceeding $3 \mathrm{TeV}$ looks sufficient to start an analysis of the meaning of the separation of the muons in the case of primary energies of $10^{7} \mathrm{GeV}$. At higher energies, for instance $10^{9}$ $\mathrm{GeV}$, the average number of muons (lower enery threshold $30 \mathrm{TeV}$ ) is close to 3 (with an accompaniement of 12 hadrons at ground level); but if we consider instead those vertical showers, inclined showers with $\Theta=70^{\circ}$ this average number of muons jumps from 3 to 7 and the hadron accompaniement (above the energy threshold) disappears. Such properties of inclined HE muon families (separation of muons, possible alignments or cluters, energy distribution) justify more extended simulations to obtain quantitative correlations with primary interaction features and identification of the primary mass. Dealing with UHE showers of $10^{11} \mathrm{GeV}$, in average only one pair of muons with energy above $300 \mathrm{TeV}$ are generated and arrive at sea level for a proton primary (with a hadron accompaniement of 21 hadrons) instead of 6 muons if $\Theta=70^{\circ}$, respectively 8 muons in the case or an iron primary nucleus. Unfortunately, such target maps would be too rare in the present detector arrays.

\section{Conclusion}

Our modest approach of the structure of the penetratig component in energetic EAS cores indicates several possibilities of new investigations based on high energy neutrinos and also muons contained inside EAS cores. In the case of neutrinos, the estimation of the energy carried by the beam in the first kilometer of crust could be used for acoustic detection whereas the nadir angle distribution of the muons generated at the antipodes could be exploited to estimate the earth core densities and the profile of the mantle. The relation of upward cascade correlated with giant showers arriving needs a very large area of detection. The satellite observation could be a solution if the threshold of detection by fluorescence could be decreased thanks to optoelectronics systems of improved resolution and better background rejection. The effect of the magnetic field on high energy muons remaining very concentrated in shower cores remains limited and the resolution of large underground arrays could be employed to distinguish heavy nuclei-air collisions in the LHC energy region to point out possible assymmetries in the fragmentation region of the interaction. A next step of simulations is in progress to delimitate the best energy thresholds of muons in muons families initiated by proton and heavy nuclei above $10^{6} \mathrm{GeV}$ to follow the energy range covered by the LHC $\sqrt{(} s)=1-15 \mathrm{TeV}$ and appreciate the interest of vertical walls with emulsion bricks like in OPERA adapted to very inclined HE muon families.

\section{References}

[1] A. de Rujula, S. L. Glashow, R. R. Wilson and G. Charpak, Physics Reports 99, No 6, 341-396 (1983).

[2] V. Berezinski and G. T. Zatsepin, Phys. Lett. 28B, 423 (1969).

[3] C. T. Hill and D. N. Schramm, Phys. Lett. 131B, 247 (1983).

[4] S. I. Nikolski and V. A. Tsarev, Kratk. Soobshch. Fiz. 1, 57 (1984).

[5] V. Berezinski et al, Yad.Fis. 43, 406 (1986).

[6] Y. Minorikawa and K. Mitsui , Europhys. Lett. 7, 77 (1988).

[7] T. Gaisser, Cosmic Rays and Particle Physics (Cambridge University Press, New York, 1990) 188-191.

[8] D. Heck, J. Knapp, J.N. Capdevielle, G. Schatz and T. Thouw, FZK A report-6019 ed. FZK The CORSIKA Air Shower Simulation Program, Karlsruhe (1998).

[9] J. N. Capdevielle et al., J. Phys. G 36, 075205 (2009).

[10] A. Cazes CNGS beam and muon identification in OPERA Ph. D. Thesis, Univ. Paris VI, 2004

[11] S. Yoshida, Cosmic Rays and Dark Matter(edited by Y. Muraki, University of Nagoya), 26 (2003).

[12] V.S. Berezinski et al., Astrophysics of Cosmic rays, 370-378,(1990) North-Holland, Elsevier, Amsterdam.

[13] S. Soula et al., Journal of Geophysics Research 116, D19103 (2011). 